\title{
Discoidin Domain Receptor-1 Gene Expression of Osteoblasts on Fibronectin Coated Titanium Disks
}

\author{
Haruhiko Tsutsumi ${ }^{1)}$, Kamolparn Pugdee ${ }^{1)}$, Tohru Hayakawa, ${ }^{2,3)}$ and Yoshimitsu Abiko ${ }^{1,3)}$ \\ 1) Department of Biochemistry and Molecular Biology, Nihon University School of Dentistry at Matsudo,Chiba, 271-8587, Japan \\ ${ }^{2}$ Department of Dental Biomaterial, Nihon University School of Dentistry at Matsudo,Chiba, 271-8587, Japan \\ ${ }^{3)}$ Research Institute of Oral Science, Nihon University School of Dentistry at Matsudo,Chiba, 271-8587, Japan \\ (Accepted for publication, December 20, 2008)
}

\begin{abstract}
Enhancement of osteoblastic adhesion and migration on a titanium (Ti) surface increases the successful rate of osseointegration in implant therapy by stimulating osteoblastic differentiation. It has been reported that Ti surfaces coated with fibronectin (FN) or GRGDSP peptide derived from FN had improved initial cell attachment and supported osteoblastic cell differentiation. However, the differences in gene expression by osteoblasts grown on FN and GRGDSP coated Ti disks have not been elucidated. Ti disks were coated with FN or GRGDSP peptide, then MC3T3-E1 osteoblastic cells were cultured on those surfaces. After 15 days, the levels of gene expression of the cells were examined. Those levels were mostly similar between the FN and GRGDSP coated disks, though that of discoidin domain receptor-1 (DDR-1) was significantly greater in cells cultured on the FN coated disks. The elevated mRNA level of the DDR-1 gene was then confirmed by reverse transcription-polymerase chain reaction (RT-PCR) and real-time PCR assays. In addition to the ability of the RGD domain to bind with integrins, FN also binds to fibrillin, proteoglycan, and collagen by their specific binding domains, thus the higher level of DDR-1 gene expression seen in our experiments may have been induced by those and not by the RGD domain. For future developing biomaterial as coating for Ti implants using synthetic peptides, binding domains other than the RGD domain in FN may be useful and important.
\end{abstract}

Key words: Osteoblast, Fibronectin, RGD peptide, DDR-1, Gene expression

\section{Introduction}

In titanium (Ti) implants, the first biological reaction at the biomaterial-tissue interface is adsorption of body fluid proteins, including extracellular matrix components ${ }^{1,2}$, and it is widely accepted that proteins in the extracellular matrix control subsequent biological responses at that interface. Some studies have reported that pre-adsorption with cell-adhesive proteins, such as fibronectin (FN), on the Ti surface improved initial cell attachment, spreading, and activity ${ }^{3,4)}$. FN is required for osteoblast survival, proliferation, osteoblast specific gene expressions, and matrix mineralization ${ }^{5}$, moreover, gene expression of МС3Т3E1 osteoblastic cells cultured on FN coated Ti surfaces has been reported, which showed an increased rate of cell attachment along with enhanced mRNA levels of bone sialoprotein (BSP) and osteomodulin (OMD) ${ }^{6}$.

Synthetic peptides with a functional domain consisting of an adhesive molecule may have some advantages for the development of biomaterial implants, as a small synthetic peptide has lower

Correspondence to: Dr. Yoshimitsu Abiko,2-870-1 Sakaecho-nishi, Matsudo, Chiba 271-8587, Japan. Department of Biochemistry and Molecular Biology, Nihon University School of Dentistry at Matsudo, Chiba 271-8587, Japan Tel : 81-47-360-9328, Fax: 81-47-360-9329 immunogenicity and a lower cost of production. Zreiqat et $\mathrm{al}^{7)}$ reported that cells derived from human bone cells cultured on peptide containing GRGDSP-coated Ti surfaces displayed significantly increased levels of osteocalcin (OC) gene expression compared to non-coated Ti surfaces. In addition, Ti implants coated with the RGD peptide from dog femoral condyles showed significantly improved mechanical fixation as well as increased bone formation around the implants ${ }^{8}$.

Short RGD-motif peptides have been designed and synthesized from amino acid sequence databases of collagens, laminin, FN, and BSP, and it has been reported that GRGDSP, a peptide derived from FN, had the highest cell attachment activity among the peptides tested ${ }^{9)}$. Furthermore, after MC3T3-E1 osteoblasts were cultured on GRGDSP-coated Ti disks, gene expression profiling demonstrated increased levels of baculoviral inhibitor of apoptosis repeat-containing gene 6 (Birc6 $)^{10)}$, BSP, and OC mRNA ${ }^{11)}$ compared with the non-coated Ti control using an Affymetrix Gene Chip. In another study, MC3T3-E1 cells were cultured on FN or GRGDSP coated Ti disks, and gene expression profiling was performed using an Affymetrix Gene Chip ${ }^{12}$. Their results showed that osteoblastic gene expression levels of cells were similar 
Haruhiko Tsutsumi et al.: DDR-1 Expression of Osteoblasts on Fibronectin

between the FN and GRGDSP coated Ti-disks, which suggested that GRGDSP coated Ti may be useful for clinical applications accelerating the osseointegration of implants. However, very little has been reported regarding the differences in the molecular based mechanisms that enhance the integrity of tissue implant interfaces between FN and GRGDSP coated Ti disks.

In the present study, in order to elucidate the usefulness of FN and GRGDSP coated Ti disks for the development of biomaterial implants, gene expression profiles other than those for osteoblastic marker genes were examined in detail using an Affymetrix Gene Chip system.

\section{Materials and Methods FN and GRGDSP peptide coating onto Ti disks}

FN and GRGDSP were coated separately onto Ti surfaces using the tresyl chloride activation method described previously ${ }^{13)}$. Briefly, Ti disks (JIS, H 4600; 99.9\% Ti mass; Furuuchi Chemical Corp. Tokyo, Japan) with a diameter of $48 \mathrm{~mm}$ were obtained and ground with 600 grit sanding paper. The polished Ti disks had a scale length of $64 \mathrm{~mm}$ and a cut value of $0.8 \mathrm{~mm}$, while the surface roughness value, as measured with a Handysurf E-30A (Tokyo Seimitsu, Tokyo), was $0.76 \pm 0.06 \mu \mathrm{m}$. The surfaces of the polished Ti disks were completely covered with tresyl chloride (Fluka, Buchs, Switzerland) and stored at $37^{\circ} \mathrm{C}$, for 2 days, then washed with water followed by water-acetone solution (50:50), dried and stored in a desiccator. Human plasma FN (Harbor Bio-Products, MA, USA) and chemically synthesized GRGDSP peptide (Harbor Bio-Products, MA, USA) were separately dissolved in phosphatebuffered saline (PBS) solution $(100 \mu \mathrm{g} / \mathrm{ml}$; pH 7.4) at a concentration of $100 \mu \mathrm{g} / \mathrm{ml}$. After ultrasonic cleaning in distilled water, GRGDSP peptide was coated onto the polished Ti disks used to examine GRGDSP coating. All tresylated Ti disks were immersed into an FN or GRGDSP/PBS solution $(100 \mu \mathrm{g} / \mathrm{ml})$ for 24 hours at $37^{\circ} \mathrm{C}$, then rinsed with doubled-distilled water, and finally dried with a gentle stream of dry air and stored in a desiccator. Non-coated Ti disks were used as a control. Before starting the cell cultures, all disks were sterilized by ultraviolet radiation.

\section{Cell culture and RNA extraction}

MC3T3-E1 osteoblastic cells were plated at a density of $5 \times 10^{4}$ cells/ $\mathrm{cm}^{2}$ on 48-mm FN or GRGDSP coated Ti disks, and noncoated control disks. The cells were cultured using Minimum Essential Medium (MEM) alpha (Gibco, Invitrogen, Carlsbad, CA), with $10 \%$ fetal calf serum, antibiotics (penicillin/ streptomycin), $50 \mu \mathrm{g} / \mathrm{ml}$ of ascorbic acid, and $5 \mathrm{mM} \beta$ glycerophosphate. The medium was changed every 2-3 days. After 15 days, total cellular RNA was isolated using an RNeasy kit (QIAGEN, CA, USA).

\section{Gene chip analysis}

Total RNA samples (100 ng) were subjected to two-cycle target labeling according to the Affymetrix instructions. Antisense complimentary RNA (cRNA) derived from double strand complimentary DNA (cDNA) was labeled in the presence of biotinylated deoxyribonucleotide triphosphate (dNTP) derivatives to produce cRNA probes. The probes were then fragmented and hybridized onto the Gene Chip Mouse Genome 430 2.0 Array. Washing and staining were performed for each sample using a Gene Chip Fluidics station 450 (Affymetrix, Santa Clara, CA). Chip performance, background levels, and the presence or absence of signals were assessed using Microarray Suite software (Affymetrix, Santa Clara, CA). Each gene chip in a given set was normalized by adjusting the probe intensities. The presence or absence of signals was re-evaluated and intensity normalization was performed across all 8 of the arrays. Data analysis was performed using the Gene Chip Expression Analysis (Affymetrix, Santa Clara, CA) and GeneSpring (Silicone Genetics, Redwood, CA) software packages.

\section{Real time and endpoint RT-PCR analyses}

Real-time PCR reactions were performed using a real-time DNA thermal analyzer (Rotor-Gene ${ }^{\mathrm{TM}}$ 6000; Corbett Life Science, Sidney, Australia) with SYBR Premix Ex Taq ${ }^{\mathrm{TM}}$ (Perfect RealTime PCR, Takara, Japan) and a Green PCR kit (Qiagen GmbH, Germany). PCR was started with an initial incubation at $95^{\circ} \mathrm{C}$ for 15 seconds to activate the Taq DNA polymerase, then performed at $95^{\circ} \mathrm{C}$ for 5 seconds and $56^{\circ} \mathrm{C}$ for 15 seconds during 40 cycles. Fluorescent signals were measured at the end of each elongation step and the beginning points of their exponential curves were determined to convert the cycle number into the amount of PCR. To calculate gene expression fold changes, the initial template concentration was derived from the cycle number at which the fluorescent signal crossed the threshold in the exponential phase of the real-time PCR reaction. The respective primer sequences were 5'-caggggaagctggactcgaa-3' (the forward primer for DDR1); 5'-tcaggcaggatcсаассас-3'(the reverse primer for DDR1), predicted size $=203 \mathrm{bp}$; 5' -atcaccatcttccaggag-3' (the forward primer for GAPDH); and 5'-atcgactgtggtcatgag-3'(the reverse primer for GAPDH), predicted size $=318 \mathrm{bp}$. Values were calculated as the mean \pm standard deviation (SD). Comparisons were made between two groups using Student's t-test.

\section{Gene chip analysis}

\section{Results}

A scatter plot showing mRNA levels expressed by MC3T3-E1 cells on the FN and GRGDSP coated Ti disks after 15 days of culture is shown in Figure 1. Most of the gene expression levels on the FN and GRGDSP coated disks were similar, though a small number of genes showed alterations. Interestingly, a 3.2-fold higher level of discoidin domain receptor-1 (DDR-1) mRNA was found on the FN coated Ti disks as compared to the GRGDSP 


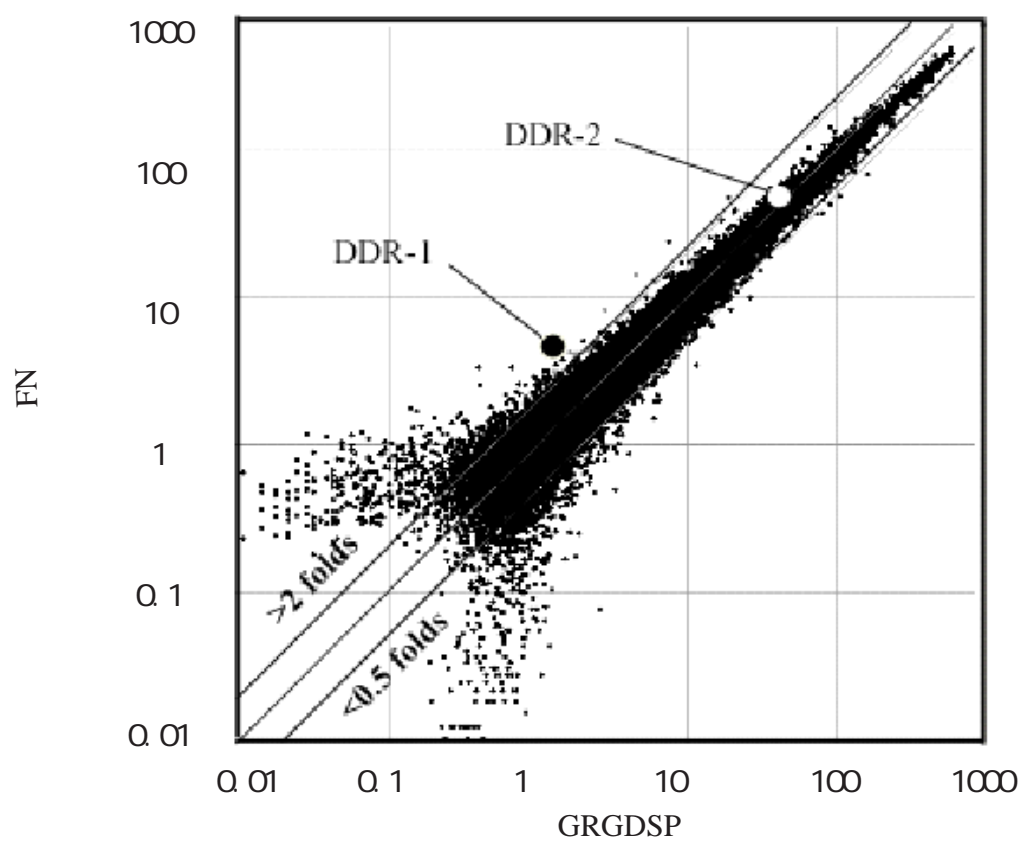

Figure 1. Scatter plot of gene expression profiles of MC3T3-E1 cells on FN and GRGDSP coated Ti disks.

The level of DDR-1 mRNA expression was greater on the FN coated disks.

DDR-1

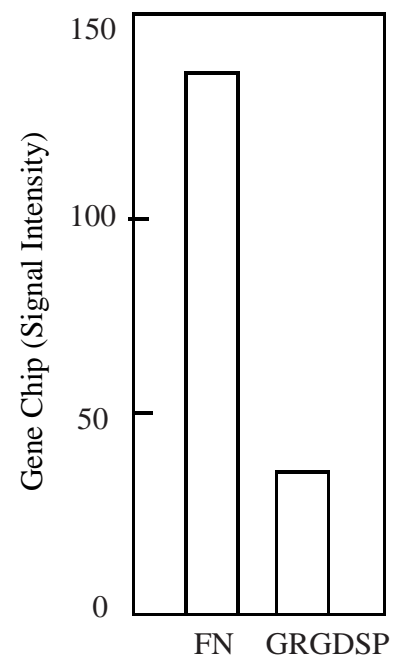

DDR-2

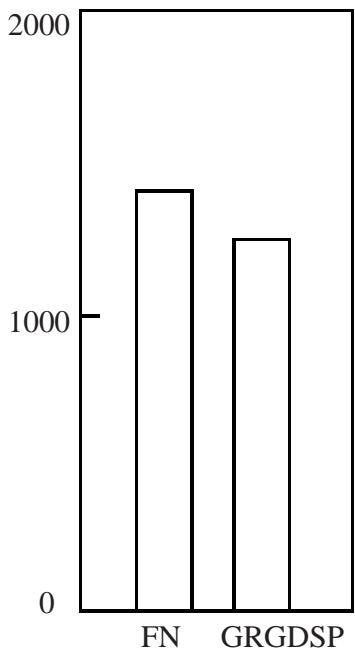

Figure 2. Raw intensity of DDR-1 and DDR-2 mRNA levels shown by Affymetrix Gene Chip analysis.

coated disks, whereas the level of mRNA of the DDR-2 gene was not significantly different (Figure 2).

\section{Endpoint RT-PCR}

To further investigate the elevated mRNA level of DDR-1 gene, end-point RT-PCR was performed, which confirmed a higher level detected from MC3T3-E1 cells cultured on FN coated Ti disks (Figure 3). In contrast, mRNA levels of GAPDH, the housekeeping

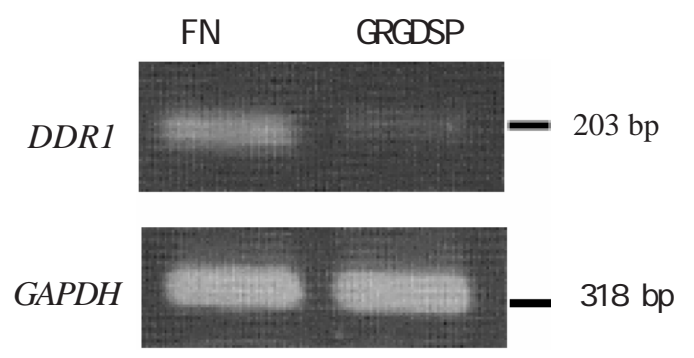

Figure 3. End-point RT-PCR analysis of DDR-1 mRNA levels. The expression of DDR-1 mRNA was greater on the FN coated compared to the GRGDSP coated Ti disks. GAPDH was used as an internal control for cDNA quality and PCR efficiency. The sizes of the amplified DNA samples were the expected numbers of base pairs for DDR-1 and GAPDH.

control, showed no differences between the FN and GRGDSP coated disks.

\section{Quantitative real-time RT-PCR}

Another experiment to determine the exact rates of enhancement of DDR-1 gene expression level was performed using real-time PCR. The results were converted to mRNA copy units and the increase in DDR-1 gene expression was shown to be significantly different between the FN coated and GRGDSP coated Ti disks (Figure 4).

The relative mRNA amounts and the ratios of DDR- 1 to GAPDH shown by gene chip analysis and real-time RT-PCR assays were calculated, and the results are summarized in Table 1. Both results of gene chip and real-time PCR are similar, and 
Haruhiko Tsutsumi et al.: DDR-1 Expression of Osteoblasts on Fibronectin

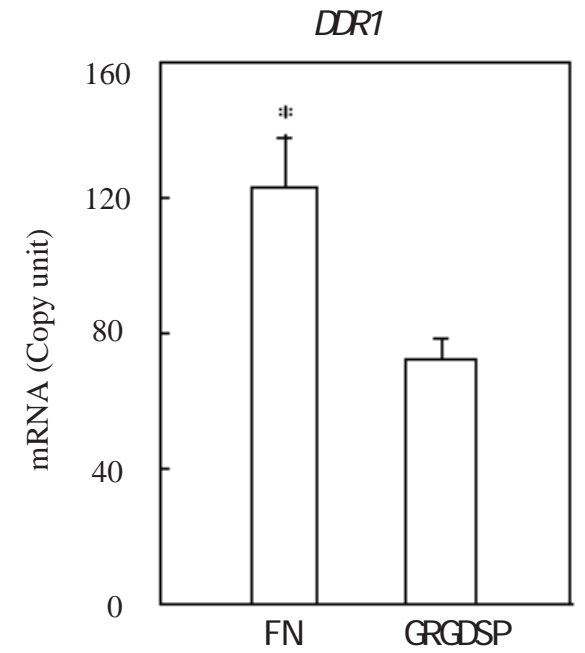

Figure 4. DDR-1 mRNA level shown by real-time PCR. The numbers of mRNA copies of MC3T3-E1 cells were significantly greater on FN coated compared to GRGDSP coated Ti disks $(* \mathrm{p}<0.05, \mathrm{n}=3)$.

DDR1 gene expression is enhanced on the FN coated Ti disks compared to GRGDSP coated Ti disks.

\section{Discussion}

It is well known that the RGD peptide sequence binds with osteoblasts via cell surface integrins ${ }^{14)}$, while other studies have shown that RGD and related peptides are able to enhance osteoblast adhesion and migration ${ }^{15,16)}$. In our previous study, MC3T3-E1 cells were cultured on both FN and GRGDSP coated Ti disks, and analyzed the gene expression profiling using an Affymetrix Gene Chip. Thus, we found that the gene expression levels of osteoblastic marker genes, including type I collagen, alkaline phosphatase, osteomodulin, osteocalcin, bone sialoprotein, osteonectin, osteopontin, core binding factor a1, osterix, and msh homeobox homolog-2 expressed by the cells were similar between the two coatings ${ }^{12)}$, which indicated that GRGDSP coated Ti could be useful for accelerating the osseointegration of implants in clinical applications. However, FN is known to link with other extracellular matrix components, such as fibrillin, proteoglycan, and collagen, through their binding domains $^{17,18)}$, thus we considered it important to compare gene expression levels using MC3T3-E1 cells cultured on FN and GRGDSP coated Ti disks.

Our results showed elevated levels of DDR-1 gene mRNA on the FN coated Ti disks. That finding, obtained using an Affymetrix Gene Chip system, was then confirmed by RT-PCR and real-time PCR assays.

The expressions of DDR genes of osteoblasts have not been previously reported. Communication between cells and their environment is mediated by specific receptors that transduce signal pathways, and important signaling receptors are often associated with tyrosine kinase, which plays important roles in a number of cellular processes, including the cell cycle, differentiation, migration, and metabolism. DDR1 and DDR2 constitute a subfamily of receptor tyrosine kinases that function as collagen receptors independent of integrin ${ }^{19,20)}$. The various functions of collagens provide not only structural support, but they also serve as bioactive molecules in cellular signaling. These two DDR genes differ in their ligand specificities, as both are activated by fibrillar collagens types, while only DDR1 can be activated by non-fibrillar collagens such as type IV collagen ${ }^{21)}$. Interestingly, DDR receptors regulate cell adhesion, migration, proliferation, and remodelling of the extracellular matrix ${ }^{22}$. In the present study, we found that DDR-1 gene expression of osteoblasts was activated when the cells were cultured on FN coated Ti disks, whereas that of DDR2 was not, suggesting that FN coating may induce the expression of non-fibrillar collagens to a greater extent than GRGDSP coating.

Collagen-induced DDR1 autophosphorylation is a cell-type= specific process that requires the secreted protein Wnt5a ${ }^{23,24)}$. Interestingly, it has been reported that Wnt5a is involved in bone formation by potentiating osteoblastic differentiation of human mesenchymal stem cells through activation of the Ror2 receptor tyrosine kinase. Taken together with our results, it is suggested that DDR-1 could play a role in osteoblastic differentiation in association with Wnt5a.

FN has several functional domains, such as the fibrillin, proteoglycan, and collagen binding domains, which may promote DDR1 gene expression. Although GRGDSP coated Ti may promote early cell attachment and matrix bone mineralization by promoting integrin binding, the fibrillin, proteoglycan, and

Table 1 Summary of gene expressions

\begin{tabular}{lcccc}
\hline Gene & Samples & Gene Chip & \multicolumn{2}{c}{ Real-time PCR } \\
& & Fold & mRNA copiy & Fold \\
\hline DDR-1 & FN & 3.2 & $123.23 \pm 14.83 *$ & 1.7 \\
& GRGDSP & & $71.79 \pm 6.89$ & \\
\hline \multirow{2}{*}{ DDR-2 } & FN & \multirow{2}{*}{1.1} & ND & ND \\
& GRGDSP & & ND & \\
\hline & *Signification different to GRDSP coated Ti disk $(* \mathrm{p}<0.01, \mathrm{n}=4)$
\end{tabular}


collagen binding domains may also be important to stimulate osteoblastic differentiation through DDR-1 gene expression. For future developing biomaterial as coating for Ti implants using synthetic peptides, binding domains other than the RGD domain in FN may be useful and important to promote early cell attachment, as well as osteoblast differentiation and matrix bone mineralization.

\section{Acknowledgements}

This work was supported by the “Academic Frontier" Project for Private Universities, a matching fund subsidy from the Ministry of Education, Culture, Sports, Science and Technology of Japan, 2007-2011.

\section{References}

1. Dean JW, Culbertson KC and D'angelo AM. Fibronectin and laminin enhance gingival cell attachment to dental implant surfaces in vitro. Int J Oral Maxillofac Implants 10(6): 721728,1995

2. Bierbaum S, Hempel U, Geissler U, Hanke T, Scharnweber D, Wenzel KW and Worch H. Modification of Ti6Al4V surfaces using collagen I, III, and fibronectin. II. influence on osteoblast responses. J Biomed Mater Res A 67(2): 431438, 2003

3. Moursi AM, Damsky CH, Lull J, Zimmerman D, Doty SB, Aota S and Globus RK. Fibronectin regulates calvarial osteoblast differentiation. J Cell Sci 109(6): 369-1380, 1996

4. Jimbo R, Sawase T, Shibata Y, Hirata K, Hishikawa Y, Tanaka Y, Bessho K, Ikeda T and Atsuta M. Enhanced osseointegration by the chemotactic activity of plasma fibronectin for cellular fibronectin positive cells. Biomaterials 28(24): 3469-3477, 2007

5. Globus RK, Doty SB, Lull JC, Holmuhamedov E, Humphries $\mathrm{MJ}$ and Damsky CH. Fibronectin is a survival factor for differentiated osteoblasts. J Cell Sci 111(10): 1385-1393, 1998

6. Pugdee K, Shibata Y, Yamamichi N, Tsutsumi H, Yoshinari M, Abiko Y and Hayakawa T. Gene expression of MC3T3E1 cells on fibronectin-immobilized titanium using tresyl chloride activation technique. Dent Mater J 26(5): 647-655, 2007

7. Zreiqat H, Akin FA, Howlett CR, Markovic B, Haynes D, Lateef S and Hanley L. Differentiation of human bonederived cells grown on GRGDSP-peptide bound titanium surfaces. J Biomed Mater Res A 64(1): 105-113, 2003

8. Schliephake H, Scharnweber D, Dard M, Rössler S, Sewing A, Meyer J and Hoogestraat D. Effect of RGD peptide coating of titanium implants on periimplant bone formation in the alveolar crest. An experimental pilot study in dogs. Clin Oral Implants Res 13(3): 312-319, 2002
9. Abiko Y, Pugdee K, Hirai T, Chu C-H, Ando T and Shibata Y. Attachment activity of RGD-motif peptides to osteoblastlike cell line MC3T3-E1. Int J Oral Med Sci 6(2): 97-99, 2007

10. Watanabe N, Pugdee K, Hayakawa T and Abiko Y. Baculoviral Inhibitor of Apoptosis Repeat-containing Gene 6 Expression in Osteoblasts on Fibronectin Derived Peptide Coated Titanium. J Jap Soc Oral Implantol 21(1): 1-6, 2008

11. Yamamichi N, Pugdee K, Chang WJ, Lee SY, Yoshinari M, Hayakawa T and Abiko Y. Gene expression monitoring in osteoblasts on titanium coated fibronectin-derived peptide. Dent Mater J 27(5): 744-750, 2007

12. Hayakawa T, Tsutsumi H, Yamamichi N and Abiko Y. Gene Expressions in Osteoblasts on Fibronectin and GRGDSP Coated Titanium. Med Biol 152(7): 286-291, 2008

13. Hayakawa T, Yoshinari M and Nemoto K. Direct attachment of fibronectin to tresyl chloride-activated titanium. J Biomed Mater Res A 67(2): 684-688, 2003

14. MacDonald DE., Markovic B, Allen M, Somasundaran P and Boskey AL. Surface analysis of human plasma fibronectin adsorbed to commercially pure titanium materials. J Biomed Mater Res 41(1): 120-130, 1998

15. Ganss B, Kim RH and Sodek J. Bone sialoprotein. Crit Rev Oral Biol Med 10(1): 79-98,1999

16. Hilbig H, Kirsten M, Rupietta R, Graf HL, Thalhammer S, Strasser S and Armbruster FP. Implant surface coatings with bone sialoprotein, collagen, and fibronectin and their effects on cells derived from human maxillar bone. Eur J Med Res 12(1): 6-12, 2007

17. Ruoslahti E. Fibronectin and its receptors. Annu Rev Biochem, 57: 375-413, 1988

18. Teti A. Regulation of cellular functions by extracellular matrix. J Am Soc Nephrol 10: S83-87,1992

19. Shrivastava A, Radziejewski C, Campbell E, Kovac L, McGlynn M, Ryan TE, Davis S, Goldfarb MP, Glass DJ, Lemke G and Yancopoulos GD. An orphan receptor tyrosine kinase family whose members serve as nonintegrin collagen receptors. Mol Cell 1(1): 25-34, 1997

20. Vogel W, Brakebusch C, Fässler R, Alves F, Ruggiero F and Pawson T. Discoidin domain receptor 1 is activated independently of beta(1) integrin. J Biol Chem 275(8): 57795784, 2000

21. Vogel W, Gish GD, Alves F and Pawson T. The discoidin domain receptor tyrosine kinases are activated by collagen. Mol Cell 1(1): 13-23,1997

22. Vogel WF, Abdulhussein R and Ford CE. Sensing extracellular matrix: an update on discoidin domain receptor function. Cell Signal 18(8): 1108-1116, 2006

23. L'Hôte CG, Thomas PH and Ganesan TS. Functional analysis of discoidin domain receptor 1 : effect of adhesion on DDR1 
Haruhiko Tsutsumi et al.: DDR-1 Expression of Osteoblasts on Fibronectin

phosphorylation. FASEB J 16(2): 234-236, 2002

24. Jönsson M and Andersson T. Repression of Wnt-5a impairs DDR1 phosphorylation and modifies adhesion and migration of mammary cells. J Cell Sci 114 (11): 2043-2053, 2001
25. Liu Y, Rubin B, Bodine PV and Billiard J. Wnt5a induces homodimerization and activation of Ror2 receptor tyrosine kinase. J Cell Biochem 105(2): 497-502, 2008 